\title{
Role of Temperament, Parenting Behaviors, and Stress on Turkish Preschoolers' Internalizing Symptoms
}

H. Melis Yavuz ${ }^{1}$, Bilge Selcuk ${ }^{1}$, Feyza Corapci ${ }^{2}$ and Nazan Aksan ${ }^{3}$

${ }^{1}$ Koc University

${ }^{2}$ Bogazici University

${ }^{3}$ University of Iowa Hospitals \& Clinics

\begin{abstract}
Child- and family-related factors that predict internalizing symptoms are understudied in preschool years and have a negative influence on children's functioning. We examined observational assessments of preschoolers' temperamental fearfulness and exuberance, mother reports of negative control, warmth, and parenting stress in a sample of 109 Turkish preschoolers. High temperamental fearfulness and low joyful/exuberant positive affectivity in addition to low warmth and high parenting stress had significant effects on internalizing symptoms. Parenting stress had both direct and indirect relations to internalizing symptoms via lower maternal warmth. When comorbid elevations in externalizing symptoms were controlled, the results were consistent with the interpretation that poor parenting practices and stress associated with the parenting role predict maladaptation in general but that the specific form of maladaptation may be best predicted by individual differences in children's temperamental characteristics. This study contributes to our understanding of risk and protective factors that predict preschoolers' internalizing symptoms with a sample from a non-Western population. These findings can guide early prevention and intervention programs to address internalizing problems in a culturally-sensitive way.
\end{abstract}

Keywords: internalizing symptoms; exuberance; fear; maternal warmth and control; parenting stress

Internalizing symptoms include developmentally inappropriate and excessive anxious/depressed affect, social withdrawal, and psychosomatic problems (Achenbach,

This study is based on the MA thesis of the first author. The data were drawn from a larger project funded in part by TUBITAK 109K016 (PI: Nazan Aksan) and Koc University. We would like to thank the parents who participated in the assessments, the graduate students who conducted the assessments (Senay Cebioglu and Irem Metin-Orta) and undergraduate students who assisted with coding used in this study (Sebnem Nasirli and Ceyda Sayali).

Correspondence should be addressed to H. Nazan Aksan, Department of Neurology, 200 Hawkins Dr, Iowa City IA 52242, USA Email: nazan-aksan@uiowa.edu 
1992; Eisenberg et al., 2001; Fite, Stoppelbein, Greening, \& Dhossche, 2008). Sad, anxious, and socially withdrawn children engage in less interactions in classroom activities, have fewer friends, report feelings of loneliness, and low self-esteem (Oland \& Shaw, 2005). The prevalence of internalizing problems in the clinical range is estimated at about 12 percent among preschoolers with samples from the USA (Briggs-Gowan, Carter, Skuban, \& Horwitz, 2001). Given that such problems interfere with children's social functioning and remain relatively stable through later years when they begin at a young age (Kagan \& Snidman, 1999; Mesman, Bongers, $\&$ Koot, 2001), prevention of internalizing symptoms requires a focus on the identification of the early risk factors such as child temperament, maternal child-rearing practices, and parenting stress (Hopkins, Lavigne, Gouze, LeBailly, \& Byrant, 2013; Mills et al., 2012; Rapee, 2001).

Studies suggest that Turkish children may have higher internalizing problems compared to Dutch and American children (Bengi-Arslan, Verhulst, van der Ende, \& Erol, 1997; Erol, Simsek, Oner, \& Munir, 2005), but they do not provide insights into possible causes of these mean-level differences across cultural contexts. Although culture has been identified as an important contextual factor that may influence the functional significance of parenting behaviors to children's adaptation (Deater-Deckard et al., 2011), relatively few studies have generated data that can speak to similarity in the pattern of relations between Western and non-Western samples (e.g., Muhtadie, Zhou, Eisenberg, \& Wang, 2013; Zhou, Lengua, \& Wang, 2009). Even fewer studies include observational or multi-method assessments of risk factors in relation to internalizing symptoms (Sagrestano, Paikoff, Holmbeck, \& Fendrich, 2003). Yet, appropriate translation of prevention and intervention programs to non-Western sociocultural contexts requires the construction of an evidence base that evaluates associations among risk/protective factors and outcomes with non-Western samples. Our overarching aim was to take this initial step by examining previously identified predictors of internalizing symptoms in a sample from Turkey, a country that rests in conjunction between Western and Eastern cultures and blends both individualistic and collectivistic values. To that end, we followed previous best practices of sampling child functioning in multiple domains including temperamental fearfulness and exuberance for their independent contributions to internalizing symptoms along with parenting stress, maternal warmth, and control.

\section{Fearful and Exuberant Temperament and Internalizing Symptoms}

Evidence consistently shows that withdrawn/fearful temperament predicts increases in internalizing symptoms both concurrently and longitudinally (e.g., Karreman, de Haas, van Tuijl, van Aken, \& Dekovic, 2010; Klein, Dyson, Kujawa, \& Kotov, 2012; Leve, Kim, \& Pears, 2005; Rapee, Schniering, \& Hudson, 2009). Fearful toddlers are more easily distressed by novelty, show higher physiological reactivity, and refrain from exploring new situations or interacting with new people (Kagan \& Snidman, 1991). Avoidance of fear-eliciting situations reduces concurrent distress but reinforces further avoidance (Dadds \& Barrett, 2001; Wilmshurst, 2009). Increased reliance on avoidance to cope with novelty can subsequently hinder the development of more appropriate coping strategies and contribute to greater internalizing symptoms (Rapee, 2001). 
In addition to anxiety, internalizing symptoms include depressed affect or low levels of positive affect (Klein et al., 2012). Children with temperamentally low exuberance (i.e., high energy positive affectivity) may be at risk for internalizing symptoms (Dougherty et al., 2013). There are two distinct approaches to measurement and conceptualization of exuberance in developmental research. Fox and colleagues refer to high intensity positive affect coupled with energetic motor approach in novel situations as exuberance (Fox, Henderson, Rubin, Calkins, \& Schmidt, 2001). Not surprisingly findings have shown that higher exuberance is a risk factor for higher externalizing and lower internalizing symptoms with this approach (e.g., Degnan, Hane, Henderson, Moas, Reb-Sutherland, \& Fox, 2011; Stifter, Putnam, \& Jahromi, 2008). By assessing joyful/exuberant positive affectivity in novel situations, these studies tend to measure low behavioral inhibition. An alternative approach to measurement of joyful/exuberant positive affectivity removes novelty cues from the eliciting context (i.e., Popping Bubbles episode). When high energy positive affect is measured in contexts not imbued with novelty cues, exuberance does not appear to be related to behavioral inhibition (Pfeifer, Goldsmith, Davidson, \& Rickman, 2002). These findings suggest that exuberance and behavioral inhibition are two distinct temperamental characteristics. Likewise, questionnaire-based assessments of low levels of positive emotionality has been linked with anxiety and depressive problems concurrently and longitudinally (Dougherty et al., 2013; Dougherty, Klein, Durbin, Hayden, \& Olino, 2010; Zhou et al., 2009). In the current study, we predicted that observational measures of exuberance assessed in contexts not imbued with novelty cues would additively contribute to internalizing symptoms over and above fearfulness/behavioral inhibition.

\section{Parenting and Internalizing Symptoms}

Several studies and reviews (e.g., Hudson \& Rapee, 2001; Letcher, Smart, Sanson, \& Toumbourou, 2009; Leve et al., 2005; McLeod, Weisz, \& Wood, 2007; Wei \& Kendall, 2014; van der Bruggen, Stams, \& Bogels, 2008) support the relations of internalizing symptoms with high levels of parental rejection (or low warmth) and control. Several mechanisms have been suggested for these associations. Physical punishment and obedience demanding behaviors without explanation decreases child autonomy and self-competence, while increasing negative arousal, helplessness, and avoidance (McLeod et al., 2007; Murray, Creswell, \& Cooper, 2009; Wood, McLeod, Sigman, Hwang, \& Chu, 2003).

Meta-analytic studies on internalizing problems point to lack of consistency in measuring parental control (e.g., McLeod et al., 2007). For example, negative control has been operationalized with definitions ranging from maternal over-control and lack of warmth to harsh and punitive discipline practices. When operationalized as the presence of parental overcontrol, overinvolvement, and lack of autonomy granting, controlling parenting has consistently predicted internalizing symptoms (Bayer, Sanson, \& Hemphill, 2006; Hudson \& Rapee, 2001; McLeod et al., 2007; Rapee, 2001). The presence of power assertive control and corporal punishment also showed concurrent and longitudinal associations with anxiety and depressive problems (Gershoff, 2002; Leve et al., 2005; Muhtadie et al., 2013), particularly with school-aged children (van der Bruggen et al., 2008). Yet, these findings appear less consistent for preschoolers (Bayer et al., 2006; Booth, Rose-Krasnor, McKinnon, \& Rubin, 1994). Together, those findings call for a careful delineation of 


\section{H. Melis Yavuz, Bilge Selcuk, Feyza Corapci et al.}

maternal control. In the current study we defined maternal negative control as maternal punitive discipline and obedience demanding behaviors.

Maternal warmth (i.e., low rejection) has also been studied as a predictor of internalizing symptoms. With the exception of a few studies (e.g., Bayer et al., 2006; Karreman et al., 2010), research has generally related lower levels of warmth to higher levels of depression (Luebbe \& Bell, 2014; McLeod et al., 2007), emotional distress (Operario, Tschann, Flores, \& Bridges, 2006), anxiety (McLeod et al., 2007), and a constellation of internalizing symptoms both concurrently (Hane, Cheah, Rubin, \& Fox, 2008; Mills et al., 2012) and longitudinally (Bayer et al., 2006; Bayer, Hastings, Sanson, Ukoumunne, \& Rubin, 2010; Kuhlman, Olson, \& Lopez-Duran, 2013). The predictive associations between early warmth and later internalizing behaviors point to the protective role of early positive parenting practices with suggested mechanisms such as improvements in child's behavioral (von Suchodoletz, Trommsdorff, \& Heikamp, 2011), emotional regulation (Davidov \& Grusec, 2006), self-competency (Bayer et al., 2010), parent-child mutual positive affectivity (von Suchodoletz et al., 2011), and stress reactivity (Kuhlman et al., 2013).

Interactions between parenting dimensions may also predict internalizing symptoms. Raudino et al. (2013) showed that high maternal warmth attenuates the relations between maternal intrusiveness and child anxiety in Western sociocultural contexts. The familiar parenting styles such as authoritarian or authoritative parenting are essentially interactive effects between parental control and parental warmth. Importantly however, research has revealed that the relations between warmth and control are not uniform across a wide range of cultural groups (Deater Deckard et al., 2011). Studies in Turkey reveal that Turkish mothers report high warmth (Nacak, Yagmurlu, Durgel \& van de Vijver, 2011; Kagitcibasi, 2010; Sen, Yavuz, \& Yagmurlu, 2013), but also high levels of control and power assertion to secure obedience (Sen et al., 2013). Recent evidence from Turkey suggests that high levels of control coupled with high parental warmth predict lower externalizing symptoms (Akcinar \& Baydar, 2014). However, the additive/interactive effects of maternal negative control and warmth on internalizing symptoms in Turkish preschoolers are largely unknown and we addressed this gap.

\section{Temperament and Parenting}

Evidence shows both temperament and parenting contribute additively and interactively to elevations in internalizing symptoms (e.g., Lengua, Wolchik, Sandler, \& West, 2000; Mills et al., 2012; Porter et al., 2005). For example, Karreman et al. (2010) showed fearful children exposed to highly controlling parenting behaviors were rated higher on concurrent internalizing problems by their parents and teachers. Gilliom and Shaw (2004) showed boys from disadvantaged families with high negative emotionality/fearfulness and exposed to negative maternal control displayed higher internalizing symptoms across preschool years. School-aged fearful children were more prone to depression when they received harsh maternal control or rejection (Colder, Lochman, \& Wells, 1997; Lengua et al., 2000; Oldehinkel, Veenstra, de Winter, \& Verhulst, 2006). Interactive effects between maternal warmth and temperament have been relatively less investigated with preschool children. The current study examined the additive and interactive effects of both temperamental fear and exuberance based on behavioral observations with 
questionnaire reports of maternal negative control and warmth in relation to preschoolers' internalizing symptoms.

\section{Parenting Stress}

Parenting stress is defined as psychological distress and negative feelings associated with the demands of the parenting role (Anthony, Anthony, Glanville, Naiman, Wanders \& Shaffer, 2005; Deater-Deckard, 1998). Data support concurrent (e.g., Anthony et al., 2005; Costa, Weems, Pellerin \& Dalton, 2006; Mills et al., 2012) and longitudinal (e.g., Bayer et al., 2006; Crnic, Gaze, \& Hoffman, 2005) links between parenting stress and child internalizing symptoms. Parenting stress has also been associated with lower warmth and harsher discipline (Anthony et al., 2005; Bayer et al., 2006; Deater-Deckard, 1998; Deater-Deckard \& Scarr, 1996). As already reviewed, these parenting dimensions predict child internalizing problems. However, evidence for an indirect role of parenting stress on child outcomes through poorer parenting practices is less consistent. Some studies report mediation (Bayer et al., 2006; Deater-Deckard, 1998) while others do not (Crnic et al., 2005; Hopkins et al., 2013). Neither the direct nor the indirect associations of parenting stress have been investigated in the Turkish cultural context and will be addressed in the current study.

\section{Present Study}

Previous studies have shown higher rates of internalizing symptoms among Turkish children compared to their Western counterparts (Bengi-Arslan et al., 1997; Erol et al., 2005). However, no study to date has investigated the sources of variability in internalizing symptoms among Turkish preschoolers from multiple domains (i.e., temperament, parenting practices, parenting stress) with multi-method assessments. Hence, an overarching aim of the current study was to examine whether the associations of these factors with internalizing symptoms were similar to those reported from Western samples. While this aim informs possible sources of variability across cultural contexts in adaptive and maladaptive processes associated with internalizing symptoms to be tested in future studies, our design did not permit a formal empirical test of such cultural differences (i.e., cultural moderation). Importantly, to ensure that the tested hypotheses concerned internalizing symptoms rather than symptom elevations in general, we tested each substantive hypothesis twice: Once without and once with statistical controls for externalizing symptoms. Thus, our inferences are informed by comorbid elevations in symptoms using a variable-centered approach.

We improved on previous studies in preschoolers in two important respects. First, going beyond assessments of fearful temperament, a robust predictor of elevations in internalizing symptoms in Western contexts, we also included energetic positive affectivity or exuberance. Second, we observed children's exuberance in contexts not imbued by novelty cues (Pfeifer et al., 2002). We would expect lower exuberance and higher fearfulness to predict higher internalizing symptoms. In addition, we considered interactive effects as children who are both high in fearfulness and low in exuberance can be particularly vulnerable to elevations in internalizing symptoms.

With respect to parenting practices, we measured both maternal negative control and warmth. Given findings regarding weaker associations of maternal control with 


\section{H. Melis Yavuz, Bilge Selcuk, Feyza Corapci et al.}

internalizing symptoms in preschool as opposed to school ages (Van der Bruggen et al., 2008), we would expect low warmth to be particularly predictive of higher internalizing symptoms. Interactive effects of these parenting dimensions on outcomes were rarely tested explicitly in Western samples. Recent research from Turkey shows control and warmth interact to predict externalizing symptoms in preschool years (Akcinar \& Baydar, 2014). Low maternal warmth coupled with high negative control may be associated with greater internalizing symptoms compared to high maternal warmth and high negative control, considering the normative emphasis on parental control in the Turkish cultural context (Sen et al., 2014).

We also examined the interactive effects of parenting with child temperament. Again, because of the normative emphasis on parental control in the Turkish context, maternal warmth may play a critical role in reducing the risks associated with fearful temperament. Interactions involving parenting dimensions and child exuberance were exploratory. Finally, we tested the effects of parenting stress on internalizing symptoms directly and indirectly, through parenting practices. Given the inconsistencies regarding indirect effects of parenting stress with Western samples, we expected higher stress to be associated with increases in internalizing symptoms as well as lower warmth and greater negative control.

\section{Method}

\section{Participants}

The participants included 118 Turkish preschoolers $\left(M_{\text {age }}=4.79\right.$ years, $S D=1.33$; range $=2.2-6.2$ years $)$ and their mothers $\left(M_{a g e}=36\right.$ years, $\left.S D=3.58\right)$. Families were recruited through three private daycare centers located in middle to high socioeconomic status (SES) neighborhoods of Istanbul, hence the sample represents socioeconomically advantaged Turkish families. Recruitment process was managed through center administrators with little to no opportunity for researchers to interact with classroom teachers who have direct contact with parents. Together, these factors did not permit us to track the percentage of parents who declined to participate or to track whether the teachers approached all parents in their classrooms. Individualized reports regarding child's relative standing on a variety of measures collected in the lab (e.g. \%-ile rank on fearfulness) were offered as an incentive to parents.

The mean age for boys $(n=68 ; M=4.65$ years, $S D=.98)$ and girls $(n=50$; $M=4.35$ months, $S D=.87)$ did not significantly differ, $F(1,117)=2.95$, NS. Majority (89 percent) were from intact families. Average number of years of education for both mothers and fathers was 15.42 years, $(S D=2.78$ for mothers, $S D$ $=2.99$ for fathers). Seventy-eight percent of the mothers and 83.5 percent of the fathers had a university degree or higher. Approximately, 67 percent of mothers and 99.1 percent of fathers were employed full- or half-time. Forty percent of families had high household income (i.e., 5000 USD per month, 2008-rate). Education and household income were significantly related and were standardized and averaged to obtain family SES.

\section{Procedure}

Mothers were observed during 2.5 to 3 -h session in a laboratory decorated like a typical living room. The assessments were conducted by two female graduate students (Es) and videotaped from behind a one-way mirror. For observed 
temperament, inter-rater reliability was established on 20 percent of the cases and coders were blind to the study hypotheses. Mothers completed questionnaires regarding warmth, negative control, parenting stress, and children's internalizing symptoms.

\section{Measures}

Parenting Stress. Mothers' parenting stress was measured with three parent domain subscales of the Parenting Stress Index (Abidin, 1997) that tapped feelings of role restriction (7-items), sense of incompetency (11-items), and relationship with spouse (7-items) based on the 101-item version. Items were rated on a 5-point Likert scale $(1=$ strongly disagree to $5=$ strongly agree $)$. All subscale scores were positively correlated ( $r$ 's $.46-.57, p<.001$ ). These scores were standardized and averaged to calculate a total parenting stress score $(\alpha=.87)$.

Maternal Warmth and Negative Control. Mothers completed the Warmth (6-items; e.g., 'My child and I have warm, intimate times together'), Punishment (5-items; e.g., 'I slap or hit my child to control his/her behavior'), and Obedience Demanding (8-items; e.g., 'I expect my child to do what he/she is told to do, without argument') subscales of the Child Rearing Questionnaire (CRQ; Paterson \& Sanson, 1999). CRQ assesses the frequency of positive (warmth) and negative control (punishment and obedience demand $)$ on a 5 -point Likert scale $(1=$ never to $5=$ always $)$ and its Turkish version has been validated (Yagmurlu \& Altan, 2010; Yagmurlu \& Sanson, 2009). Alphas were .74 for warmth and .73 for negative control.

Child Temperament. Children's temperamental fearfulness (modified Spider) and exuberance (Popping Bubbles) were assessed with episodes from the Laboratory Temperament Assessment Battery (Goldsmith \& Rothbart, 1996; Pfeifer et al., 2002). In Popping Bubbles, the child was introduced to a bubble gun and told that she could blow up the bubbles in any way that s/he wished (e.g., by jumping or touching with finger). The episode lasted for 90 -s and mothers were not in the room. In the modified Spider episode, the child was introduced to a remotecontrolled truck outfitted with a scary mask, a hat, and a wig. After the initial exposure to the object, the Es gave 5 systematic prompts to the child, mainly to approach, touch or play with the toy (e.g., 'You can come closer to see it better', 'You can touch it if you want'). The episode lasted for $90-$ s and mothers stayed in the room but were asked to be busy with questionnaires.

Exuberance Coding and Data Reduction. Coders noted the presence-absence of smile, laughter, positive vocalizations, high (e.g., jumping to pop bubbles), and low energy (e.g., waiting for bubbles to land on hand) movements in 5-s segments; latency to the first smile, high, and low energy movements; peak intensity of smiling (0-3 scale) every 30-s. Inter-rater reliabilities for the exuberance episode (Kappa) ranged from .86 to .90 .

All presence-absence judgments were converted to relative frequency scores to capture the total duration of smiling, laughing, positive vocalizations, high and low energy movements. The latency scores were reversed, and the peak intensity scores were averaged. The intercorrelations of these measures were in the expected direction and ranged from .05 to .63 ( 8 out of 10 were statistically significant), average 
$r=.31$, each was $z$-scored, reversed when appropriate, and averaged to compute the exuberance composite $(\alpha=.76)$.

Fearfulness Coding and Data Reduction. Coders noted latency to approach and touch the toy, responses to standard prompts to approach $(0=$ acting before the prompt to $4=$ withdrawal/escape from situation) and maternal proximity seeking $(0=$ distant to $2=$ arm's reach or less $)$. In addition to responses immediately following prompts, children's distress reactions in the time that elapsed between each prompt was noted. Distress could occur in any of three modalities including facial (e.g. fearful facial expression), vocalizations (e.g., 'scary!' or crying), or bodily responses (e.g., freezing). Inter-rater reliabilities for the fearfulness episode (Kappa) ranged from .70-1.00.

Intercorrelations among the reversed latency scores, approach-withdrawal responses after each prompt, and proximity seeking with the mother were significant ( $r$ 's ranged from .25 to .66, $p<.01$ ); hence these scores were standardized and averaged to create the composite fearfulness score $(\alpha=.79)$.

Child Internalizing Symptoms. Child internalizing symptoms were measured with the Child Behavior Checklist, CBCL/2-3 (Achenbach, Edelbrock, \& Howell, 1987). The subscale has 36 items rated on a 3-point Likert scale $(0=$ not true, $2=$ often true), and has been validated with Turkish samples (Erol et al., 2005). Alpha was satisfactory in this sample $(\alpha=.82)$. We also included externalizing subscale in analyses to examine the extent to which the inferences reflected processes about internalizing symptoms specifically rather than comorbid elevations in symptoms. The two subscales were moderately correlated, $r=.48, p<.001$. All analyses were conducted on both the raw and the residualized internalizing symptom score. The latter was obtained by residualizing the raw externalizing score from internalizing and using the standardized residual in the analyses.

\section{Statistical Analyses}

The analyses proceeded in three stages. First, descriptive statistics and intercorrelations were examined in preliminary analyses. Second, regressions tested the main and interactive effects of temperament and parenting while controlling for demographic variables (i.e., child age, sex, SES). Third, path analyses tested hypothesized links. Model fitting began with a highly constrained baseline/null model, that constrained all substantive links to zero and subsequent models progressively introduced hypothesized links to permit nested model comparisons. All analyses were conducted twice (shown in Tables and Figures), with and without statistical controls for externalizing symptoms. We report discrepancies in inferences between the two sets of analyses in text.

Model fitting analyses were conducted with AMOS using maximum likelihood (Arbuckle, 2006). Adequacy of overall fit was examined with model chi-square and the following fit indices and their 90 percent Confidence Interval (CI): Root Mean Square Error of Approximation (RMSEA), the Comparative Fit Index (CFI), and the Expected Cross-Validation Index (ECVI). When the model is well-specified in the population of interest, chi-square is expected to be non-significant, RMSEA lower than .08, and CFI to be at or higher than .90, values of ECVI to be less than 
its value in the saturated model. Indirect effects from the final model with adequate fit were tested with empirical bootstrapping.

\section{Results}

\section{Preliminary Analyses}

Table 1 presents the descriptive statistics and intercorrelations among all the variables for $\mathrm{N}=109$. Data from 9 children were excluded as scatterplots revealed these cases to be influential. Comparisons showed that the excluded cases were rated higher on raw internalizing $(p=.024)$, marginally higher on residualized internalizing $(p=.051)$, and marginally lower on SES variable $(p=.083)$ with Mann Whitney $U$ tests. Non-parametric tests were conducted as homogeneity of variance assumption was severely violated in all three cases, $\min F(1,115)=7.03, p=.009$. None of the other variables differed between these two groups, $p$ 's $>.138$ with oneway ANOVAs. There was no missing data on variables shown in Table 1 among the 109 participants included in the analyses.

Table 1 data indicate that children's internalizing symptoms were generally low, and mothers were high in warmth and low in negative control. These statistics and histograms indicated acceptable variability in all variables. SES was not but child age was significantly negatively associated with internalizing symptoms. Girls $(M=.15, S D=.11)$ were marginally more fearful than boys $(M=-.14, S D=.09)$ $(F(1,108)=3.86, p=.052)$ but did not show differences on other variables. Maternal warmth and negative control were negatively associated. Parenting stress was associated with lower warmth, higher negative control, and internalizing symptoms. Internalizing symptoms were related to lower exuberance, higher fear (both observed), lower self-reported maternal warmth but unrelated to negative control. Children's exuberance and fear were not associated with each other or with warmth and negative control. In contrast, residualized internalizing symptoms were not correlated with maternal warmth and marginally correlated with parenting stress but continued to show significant correlations with exuberance and fearfulness.

\section{Hierarchical Regression Analyses}

Regressions tested the contribution of demographic, observed temperament, maternal warmth and negative control, parenting stress in addition to temperament-bytemperament, parenting-by-parenting, and temperament-by-parenting interaction effects. These regressions were conducted twice: without and with statistical controls for externalizing symptoms. In the first step, demographic variables (child age, sex, and SES) were entered, and in the second regression controlling for comorbid elevations in symptoms, externalizing scores were added in this first step. In the second step, the main effects of fearfulness, exuberance, maternal warmth, negative control, and parenting stress were entered. In the third step, two-way interactions were evaluated. None of the two-way interactions were significant. The results did not indicate multicollinearity (e.g. Variance Inflation Factor $>2.5$, tolerance values $<.40$; Allison, 1991). However, to ensure that loss of power in the larger simultaneous regression did not lead us to overlook significant interactions of a priori interest, we also tested the interactions in smaller batches (temperament-by-temperament, parenting-by-parenting, and fearfulness-by-parenting, exuberance-by-parenting). Statistical power for interactions was at least .75 for a medium effect size of $f^{2}=.05$. 
118 H. Melis Yavuz, Bilge Selcuk, Feyza Corapci et al.

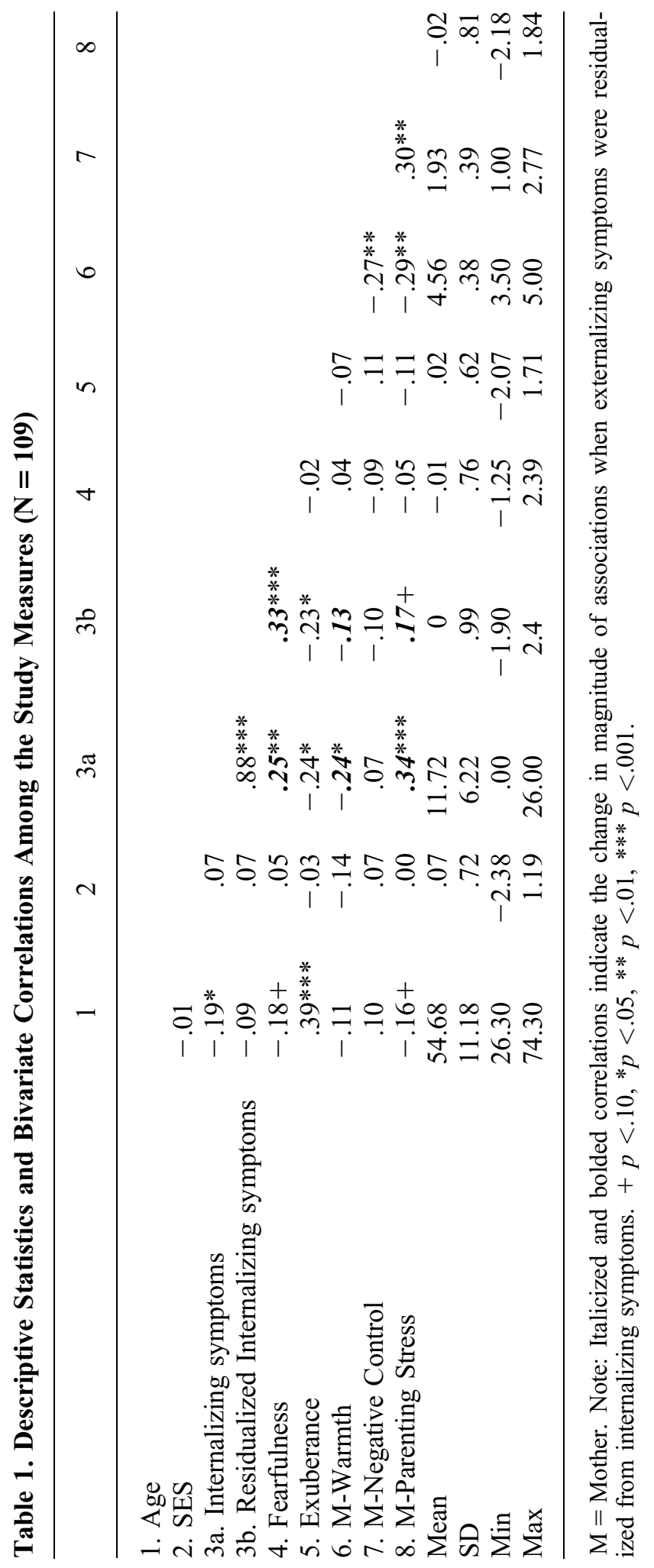


Table 2. Standardized Regression Coefficients and $\boldsymbol{R}$-square Change in the Prediction of Internalizing Symptoms With and Without Controlling for Comorbid Elevations in Externalizing Symptoms

\begin{tabular}{|c|c|c|c|c|}
\hline \multirow[b]{2}{*}{ Step \# Predictors } & \multicolumn{2}{|c|}{$\begin{array}{l}\text { Internalizing } \\
\text { Symptoms }\end{array}$} & \multicolumn{2}{|c|}{$\begin{array}{c}\text { Residualized } \\
\text { Internalizing } \\
\text { Symptoms }\end{array}$} \\
\hline & $\beta$ & $R$-sqch & $\beta$ & $R$-sqch \\
\hline Step-1 & & .05 & & $.26 * *$ \\
\hline Sex & .04 & & .12 & \\
\hline Age & -.02 & & -.06 & \\
\hline SES & .02 & & .04 & \\
\hline Externalizing Symptoms & $\mathrm{N} / \mathrm{A}$ & & $.48 * *$ & \\
\hline Step-2 & & $.21 * *$ & & $.15^{* *}$ \\
\hline Fearfulness & $.25 * *$ & & $.29 * *$ & \\
\hline Exuberance & $-.20^{*}$ & & $-.20 *$ & \\
\hline M-Negative Control & -.07 & & -.12 & \\
\hline M-Warmth & $-.21 *$ & & -.12 & \\
\hline Parenting Stress & $.27 *$ & & $.17+$ & \\
\hline Step-3 & & .04 & & .03 \\
\hline Fearfulness $\times$ Exuberance & -.02 & & -.02 & \\
\hline M Warmth $\times$ Negative Control & .03 & & .03 & \\
\hline Fearfulness $\times$ M-Warmth & -.03 & & -.03 & \\
\hline Fearfulness $\times$ M-Negative Control & .15 & & .15 & \\
\hline Exuberance $\times$ M-Warmth & .03 & & .03 & \\
\hline Exuberance $\times$ M-Negative Control & .07 & & .07 & \\
\hline
\end{tabular}

$* p<.05,{ }^{* *} p<.01$ or better. N/A = not applicable.

The inferences were no different than what is shown in Table 2 with simultaneous entry of all interactions. Exuberance and fearfulness significantly and parenting stress marginally significantly added to prediction. When externalizing symptoms were not controlled, both maternal warmth and parenting stress significantly predicted internalizing symptoms.

\section{Model Fitting Analyses}

All models and fit statistics are presented in Table 3. These analyses began with the construction of a baseline, null model that constrained all substantive links to zero and freely estimated intercorrelations among the temperament and parenting dimensions, and age was allowed to predict residualized internalizing symptoms. The fit of this model was highly inadequate. Model-1a tested the proximal effects of fearfulness and exuberance on residualized internalizing symptoms. Both paths were significant, and overall fit improved over the baseline model, $\Delta \chi^{2}(2, \mathrm{~N}=109)$ $=19.32, p<.01$. Model- $1 \mathrm{~b}$ tested the proximal effects of maternal warmth and negative control on symptoms. Only the path from maternal warmth to symptoms was 
Table 3. Overall Model Fit Statistics

\begin{tabular}{lrccccccc}
\hline & & & & \multicolumn{3}{c}{$\begin{array}{c}\text { Percent } \\
\text { 90 CI of }\end{array}$} \\
Model & $d f$ & $\chi^{2}$ & $p$ & RMSEA & RMSEA & ECVI & Percent \\
90 CI of & ECVI & CFI \\
\hline Baseline Model & 14 & 55.33 & .000 & .165 & $.12-.212$ & .71 & $.55-.95$ & .42 \\
Model-1a & 12 & 36.55 & .000 & .138 & $.073-.190$ & .60 & $.48-.79$ & .67 \\
Model-1b & 10 & 31.98 & .000 & .143 & $.089-.200$ & .58 & $.47-.76$ & .73 \\
Model-2 & 8 & 15.83 & .045 & .095 & $.01-.164$ & .51 & $.45-.65$ & .89 \\
Model-3 & 7 & 12.75 & .078 & .083 & $.00-.162$ & .51 & $.46-.64$ & .91 \\
\hline
\end{tabular}

Note: Saturated model ECVI=.52. RMSEA = Root Mean Square Error of Approximation; $\mathrm{CI}=$ Confidence Interval; $\mathrm{CFI}=$ Comparative Fit Index; ECVI $=$ Expected Cross-Validation Index.

significant, but the addition of these paths improved overall fit marginally significantly over Model-1a, $\Delta \chi^{2}(2, \mathrm{~N}=109)=4.57, p=.10$. Overall fit of Model-1b remained significant indicating the inadequacy of this simple additive model. In additional models, we tested the indirect effect of parenting stress on residualized internalizing symptoms via parenting behaviors (Model-2). Both paths from stress to maternal warmth and negative control were significant, and overall fit improved over Model-1b, $\Delta \chi^{2}(2, \mathrm{~N}=109)=16.15, p<.001$, indicating that parenting behavior may mediate the effects of parenting stress on symptoms. The overall fit of Model-2 remained significant indicating the indirect-effects only model was inadequate. When the direct effect of parenting stress on residualized internalizing symptoms was added in Model-3, overall fit improved marginally over Model-2, $\Delta \chi^{2}(1, \mathrm{~N}=109)=3.08, p=.079$. Overall fit of model-3 was marginally significant and CFI exceeded .90 .

Indirect Effects. The indirect effects in the best-fitting Model-3 depicted in Figure 1 were estimated with empirical bootstrapping (on 5000 random samples) and associated bias corrected 95 percent confidence intervals (Arbuckle, 2006). The indirect effects of child age $\left(\beta=-.007 ; \beta_{\text {standard error }}=.004\right.$; percent $95 \mathrm{CI}=-.015-.001 ; p$ $<.02$ ) on residualized internalizing behaviors (via child exuberance) was significant. The indirect effect of parenting stress on residualized internalizing behaviors via both control and warmth was not significant $\left(\beta=.003 ; \beta_{\text {standard error }}=.049\right.$; percent $95 \mathrm{CI}=-.10-.10$; NS). However, this indirect effect via maternal warmth was marginally significant $\left(\beta=.045 ; \beta_{\text {standard error }}=.036\right.$; percent $95 \mathrm{CI}=-.007-.143$; $p<.10)$.

Effects of Comorbid Elevations on Externalizing Symptoms to Inferences. When models shown in Table 3 were refitted to data without residualizing internalizing symptom ratings for comorbid elevations in externalizing symptoms, the inferences were generally similar. However, significance of nested likelihood ratio chi-square statistics was at the conventional alpha-level or better, supporting greater confidence in the inferences. Specifically, temperament-only and parenting-and-temperament model (i.e., Model-1a vs Model-1b), the direct effect of stress in Model-3 (Model-3 


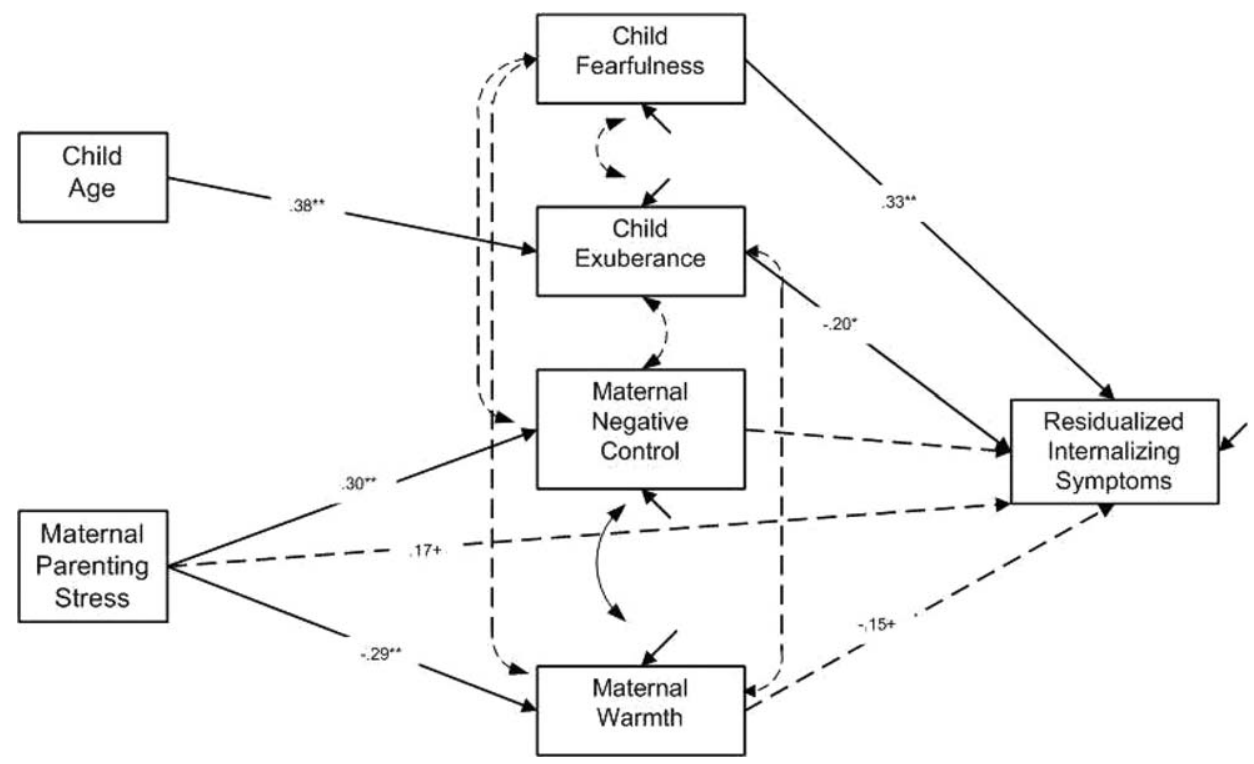

Figure 1. Standardized Path Coefficients for Model 3 with Residualized Internalizing Symptoms.

Note: Dashed lines indicate non-significant paths. $+p<.10 * p<.05, * * p<.01$ or better.

vs. Model-2 comparison) and its indirect effect via maternal warmth on symptoms were all significant at the conventional alpha.

\section{Discussion}

Previous research suggests prevalence of internalizing problems may be higher among Turkish preschoolers (e.g., Erol et al., 2005). However, the operation of risk and protective factors for internalizing symptoms using multiple domains of assessments with multiple methods has not been investigated in this population. Our overarching aim was to examine whether associations of risk factors with symptoms identified with Western samples would show a similar pattern with Turkish preschoolers drawing on direct behavioral observations of temperament and mother reports of parenting and stress. To ensure that inferences were specific to internalizing symptoms we statistically controlled for comorbid elevations in externalizing symptoms in all analyses.

Results showed that fearful children, as judged by observers, were rated higher on internalizing symptoms. Exuberance, when measured as joyful/exuberant positive affectivity in non-novel situations, predicted lower levels of internalizing symptoms. Rather than a fearfulness-by-exuberance interaction, our findings supported an additive-effects model for temperament and symptoms. Both findings were similar to those reported in Western samples regarding fearfulness (e.g. Karreman et al., 2010; Rapee et al., 2009), and positive affectivity (e.g., Dougherty et al., 2010; Zhou, 2009), indicating cross-cultural similarities in temperament-internalizing associations. Furthermore, these effects held when comorbid elevations in externalizing symptoms were statistically controlled. 


\section{H. Melis Yavuz, Bilge Selcuk, Feyza Corapci et al.}

Another finding supporting cross-cultural similarity (e.g., Bayer et al., 2006; Hane et al., 2008; Kuhlman et al., 2013; Mills et al., 2012; Siu, 2008) was that maternal warmth predicted lower levels of internalizing symptoms. Our data appears consistent with earlier suggestions that children who lack the experience of maternal warmth may have difficulty regulating their anxious and depressive affect and thus show increased internalizing symptoms (e.g., Bayer et al., 2010; Kuhlman et al., 2013). While the strength of the association between warmth and internalizing diminished when externalizing symptoms were residualized, the path coefficient remained marginally significant in the final model.

Negative control was measured as punitive discipline and obedience demands without explanation. Others have suggested that this constellation of behaviors increases internalizing symptoms by decreasing positive self-schemas, self-competence, and increasing negativity affectivity and helplessness (McLeod et al., 2007; Wood et al., 2003). Contrary to these predictions yet similar to studies with preschoolers (Booth et al., 1994; Mills et al., 2012), our results showed that negative control did not predict internalizing symptoms of Turkish preschoolers. Thus, our findings appear more consistent with the view that negative effects of parental control on internalizing symptoms may be more pronounced in school years when autonomy development becomes a more salient developmental task (van der Bruggen et al. 2008). Alternatively, it was unrelated to internalizing symptoms because of the normative emphasis on control in the Turkish context (Sen et al., 2013).

Our findings did not support parenting-by-temperament interactions. Previous research has mainly investigated the interactions of parental control and fearful temperament (e.g., Karreman et al., 2010) which do not replicate consistently (Kiff, Lengua, \& Zalewski, 2011). Inconsistencies may reflect differences in the operational definitions of power assertion, intrusiveness, and related constructs. It is also possible that both the variation in modes of measurement for these constructs (questionnaire vs. observation) and context (laboratory vs. home) account for these inconsistencies.

Our findings showed that parenting stress was associated with both decreased maternal warmth and increased negative control. However, the direct and indirect effects of stress on internalizing symptoms were generally weak when comorbid externalizing symptoms were statistically controlled. Nevertheless, our results without residualizing were more consistent with studies indicating stress has both direct and indirect roles on internalizing symptomology (e.g., Costa et al., 2006; Crnic et al., 2005) via decreased positive parenting (e.g., Mills et al., 2012), rather than increased negative parenting (e.g., Bayer et al., 2006; Hopkins et al., 2013). It is also possible that the effects of parenting stress on internalizing symptoms is a multi-chain causal process: only to the extent that parenting stress increases maternal psychopathology such as anxiety and depression, it has adverse effects on parenting, which in turn impacts internalizing symptoms (e.g., Bayer et al., 2006; Hopkins et al., 2013). Future studies would benefit from including maternal psychopathology when delineating the influences of parenting stress on internalizing symptoms.

Comorbidity Issues. Controlling for comorbid elevations in externalizing symptoms was substantively informative. The pattern of differences in findings both without and with statistical controls indicated that: a) the associations of observed exuberance and fearfulness with internalizing were generally unaffected by statistical 
controls; b) in contrast, associations of mother reported parenting and stress with internalizing were generally weakened when externalizing symptoms were controlled. This pattern has mutually non-exclusive implications for future research. First, observational measures may permit greater specificity in inferences compared to questionnaire measures, re-iterating the importance of greater reliance on these methods. Second, there may be greater specificity in the associations of temperamental reactivity but less specificity in the associations of parenting and parenting role-related stress with elevations in different symptom clusters. In other words, poor parenting practices and parenting role-related stress may be associated with child maladaptation in general but the specific form of maladaptation may be best predicted by individual differences in temperament (Cicchetti \& Rogosch, 1996).

Culturally Specific Processes. The pattern of associations we observed in this sample do not suggest the presence of a strong pattern that supports culturally specific processes in predicting risk for internalizing symptoms distinct from Western samples. However, our design and analyses did not permit a formal comparison of the extent to which cultural context may influence risk and protective processes (i.e., culture moderating associations among substantive factors). There may be at least four reasons for this: (1) our sample was generally socioeconomically advantaged, and thus atypical of broad segments of the Turkish population, (2) any differences in cultural processes may be fast becoming very subtle in an increasingly global world that necessitates reliance on larger sample sizes to detect small differences, (3) in order for cultural differences in risk processes to become apparent, greater reliance on observational measures of key constructs are necessary as this methodology is less likely to be influenced by the difficulty of establishing measurement equivalency with regard to item-meaning across cultures, and (4) magnitude of cultural differences in risk processes may be larger and thus more easily detectable when risk is also assessed at macro-levels such as the public health systems, community/neighborhood factors, and school systems (Bronfenbrenner, 1986); and the substantively most important cultural difference may concern the association of these macro-level factors with micro-level risk processes that directly bear on children's adaptation.

\section{Limitations and Conclusions}

First, cross-sectional design weakens our confidence about the direction of influences. Second, our sample was middle-to-high SES thus generalizability to broader segments of the Turkish population may be limited. Third, all variables other than temperament were obtained with maternal reports. Such shared method variance may have made the indirect effect of parenting stress on internalizing appear stronger than it may be in the population. Fourth, while statistical power was adequate to detect moderate-size interaction effects, larger samples would increase our confidence that those interaction effects are negligible. Future studies with observed parenting measures in samples from lower SES backgrounds are required to replicate these findings.

Despite these limitations, this study speaks to risk and protective factors for internalizing symptoms in Turkish preschoolers with multi-domain and multi-method assessments. Unlike most previous studies, we examined the interplay of these factors on internalizing symptoms by statistically controlling for comorbid elevations in 
externalizing symptoms. The results showed that temperamental fearfulness and parenting stress acted as risk factors, temperamental exuberance and maternal warmth acted as protective factors; and majority of these effects had additive rather than multiplicative effects on symptom elevations. There was some evidence for an indirect effect of parenting stress on children's internalizing symptoms as well, possibly mediated by decreased maternal warmth. One promising implication of these findings is that prevention/intervention programs should invest in stress management for parents both generally and specifically with respect to their parenting role. Such investments may permit parents to be both more positively inclined toward changing their habitual parental responses in favor of more adaptive ones and more aware of the effects of their stress on their children's well-being and future adjustment. A second implication of our findings is that intervention and prevention programs developed in Western contexts may also prove useful in the Turkish sociocultural context, especially for families from socioeconomically advantaged backgrounds.

\section{References}

Abidin, R. (1997). Parenting stress index: A measure of parent-child system. In C. Zalaquett \& R. Wood (Eds.), Evaluating stress: A book of resources (pp. 277-291). Lanham, MD: Scarecrow Press.

Achenbach, T. M. (1992). Manual for the child behavior checklist/2-3 and 1992 profile. Burlington: University of Vermont, Department of Psychiatry.

Achenbach, T. M., Edelbrock, C., \& Howell, C. T. (1987). Empirically-based assessment of the behavioral/emotional problems of 2- and 3-year-old children. Journal of Abnormal Child Psychology, 15, 629-650. doi: 10.1007/BF00917246

Allison, P. D. (1991). Multiple regression: A premier. Thousand Oaks, CA: Pine Forge Press.

Anthony, L. G., Anthony, B., Glanville, D. N., Naiman, D. Q., Waanders, C., \& Shaffer, S. (2005). The relationships between parenting stress, parenting behavior and preschoolers' social competence and behavior problems in the classroom. Infant and Child Development, 14, 133-154. doi: 10.1002/icd.385

Akcinar, B., \& Baydar, N. (2014). Parental control is not unconditionally detrimental for externalizing behaviors in early childhood. International Journal of Behavioral Development, 38, 118-127. doi: 10.1177/0165025413513701

Arbuckle, J. L. (2006). Amos (Version 7.0) [Computer Program]. Chicago: SPSS.

Bayer, J., Hastings, P., Sanson, A., Ukoumunne, O., \& Rubin, K. (2010). Predicting midchildhood symptoms: A longitudinal community study. International Journal of Mental Health Promotion, 12, 16-28. doi: 10.1080/14623730.2010.9721802

Bayer, J., Sanson, A., \& Hemphill, S. (2006). Parent influences on early childhood internalizing difficulties. Journal of Applied Developmental Psychology, 27, 542-559. doi: 10.1016/j.appdev.2006.08.002

Bengi-Arslan, I., Verhulst, F.C., van der Ende, J., \& Erol, N. (1997). Understanding childhood (problem) behaviors from a cultural perspective: Comparison of problem behaviors and competencies in Turkish immigrant, Turkish and Dutch children. Social Psychiatry and Psychiatric Epidemiology, 32, 477-484. doi: 10.1007/BF00789143

Booth, C., Rose-Krasnor, L., McKinnon, J. A., \& Rubin, K. (1994). Predicting social adjustment in middle childhood: The role of preschool attachment security and maternal style. Social Development, 3, 189-204. doi: 10.1111/j.1467-9507.1994.tb00040.x

Briggs-Gowan, M., Carter, A., Skuban, E., \& Horwitz, S. (2001). Prevalence of socialemotional and behavioral problems in a community sample of 1- and 2- year old children. Journal of American Academy of Child and Adolescent Psychiatry, 40, 811-819. doi: 10.1097/00004583-200107000-00016

Bronfenbrenner, U. (1986). Ecology of the family as a context for human development: Research perspectives. Developmental Psychology, 22, 723-742. doi: 10.1037/00121649.22.6.723 
Cichetti, D., \& Rogosch, F. A. (1996). Equifinality and multifinality in developmental psychopathology. Development and Psychopathology, 8, 597-600.

Colder, C., Lochman, J., \& Wells, K. (1997). The moderating effects of children's fear and activity level on relations between parenting practices and child symptomatology. Journal of Abnormal Child Psychology, 25, 251-263. doi: 10.1023/A:1025704217619

Costa, N., Weems, C., Pellerin, K., \& Dalton, R. (2006). Parenting stress and childhood psychopathology: An examination of specificity to internalizing and externalizing symptoms. Journal of Psychopathology and Behavioral Assessment, 28, 113-122. doi: 10.1007/ s10862-006-7489-3

Crnic, K. A., Gaze, C., \& Hoffman, C. (2005). Cumulative parenting stress across the preschool period: relations to maternal parenting and child behaviour at age 5. Infant and Child Development, 14, 117-132. doi: 10.1002/icd.384

Dadds, M., \& Barrett, P. (2001). Practitioner review: Psychological management of anxiety disorders in childhood. Journal of Child Psychology and Psychiatry, 42, 999-1011. doi: 10.1111/1469-7610.00798

Davidov, M., \& Grusec, J. (2006). Untangling the links of parental responsiveness to distress and warmth to child outcomes. Child Development, 77, 44-58. doi: 10.1111/j.14678624.2006.00855.x

Deater-Deckard, K. (1998). Parenting stress and child adjustment: Some old hypotheses and new questions. Clinical Psychology: Science and Practice, 5, 314-332. doi: 10.1037/ a 0025120

Deater-Deckard, K., Lansford, J., Malone, P. S., Alampay, L., Sorbring, E., Bacchini, D., et al. (2011). The association between parental warmth and control in thirteen cultural groups. Journal of Family Psychology, 25, 790-794. doi: 10.1037/a0025120

Deater-Deckard, K., \& Scarr, S. (1996). Parenting stress among dual-earner mothers and fathers: Are there gender differences? Journal of Family Psychology, 10, 45-59. doi: 10.1037/0893-3200.10.1.45

Degnan, K., Hane, A., Henderson, H., Moas, L., Reeb-Sutherland, B., \& Fox, D. (2011). Longitudinal stability of temperamental exuberance and social-emotional outcomes in early childhood. Developmental Psychology, 47, 765-780. doi: 10.1037/a0021316

Dougherty, L., Klein, D., Durbin, E., Hayden, E., \& Olino, T. (2010). Temperamental positive and negative emotionality and children's depressive symptoms: A longitudinal prospective study from age three to age ten. Journal of Social and Clinical Psychology, 29, 462-488. doi: 10.1521 jscp.2010.29.4.462

Dougherty, L., Tolep, M., Bufferd, S., Olino, T., Dyson, M., Traditi, J., et al. (2013). Preschool anxiety disorders: Comprehensive assessment of clinical, demographic, temperamental, familial, and life stress correlates. Journal of Clinical Child \& Adolescent Psychology, 42, 577-589. doi: 10.1080/15374416.2012.759225

Eisenberg, N, Cumberland, A., Spinard, T. L., Fabes, S. R., Shephard, S.A., Reiser, M., et al. (2001). The relation of regulation and emotionality to children's externalizing and internalizing problem behavior. Child Development, 72, 1112-1134. doi: 10.1111/1467-8624.00337

Erol, N., Șimsek, Z., Öner, Ö., \& Münir, K. (2005). Behavioral and emotional problems among Turkish children at ages 2 to 3 years. Journal of American Academy of Child and Adolescent Psychiatry, 44, 80-87. doi: 10.1097/01.chi.0000145234.18056.82

Fite, P. J., Stoppelbein, L., Greening, L., \& Dhossche, D. (2008). Child internalizing and externalizing behavior as predictors of age at first admission and risk for repeat admission to a child inpatient facility. American Journal of Orthopsychiatry, 78, 63-69. doi: 10.1037/0002-9432.78.1.63

Fox, N., Henderson, H., Rubin, K., Calkins, S., \& Schmidt, L. (2001). Continuity and discontinuity of behavioral inhibition and exuberance: Psychophysiological and behavioral influences across the first four years of life. Child Development, 72, 1-21. doi: 10.1111/ $1467-8624.00262$

Gershoff, E. (2002). Corporal punishment by parents and associated child behaviors and experiences: A meta-analytic and theoretical review, and theoretical review. Psychological Bulletin, 128, 539-579.doi: 10.1037/0033-2909.128.4.539

Gilliom, M., \& Shaw, D. (2004). Codevelopment of externalizing and internalizing problems in early childhood. Development and Psychopathology, 16, 313-333. doi:10.1017/ S0954579404044530 
Goldsmith, H. H., \& Rothbart, M. K. (1996). The Laboratory Temperament Assessment Battery (Lab-TAB): Locomotor version 3.0. Technical Manual, Department of Psychology, University of Wisconsin, Madison, WI.

Hane, A. A., Cheah, C., Rubin, K. H., \& Fox, N. A. (2008). The role of maternal behavior in the relation between shyness and social reticence in early childhood and social withdrawal in middle childhood. Social Development, 17, 795-811. doi: 10.1111/j.14679507.2008.00481.x

Hopkins, J., Lavigne, J., Gouze, K., LeBailly, S., \& Bryant, F. (2013). Multi-domain models of risk factors for depression and anxiety symptoms in preschoolers: Evidence for common and specific factors. Journal of Abnormal Child Psychology, 41, 705-722. doi: 10.1007/s10802-013-9723-2

Hudson, J., \& Rapee, R. (2001). Parent-child interactions and anxiety disorders: An observational study. Behaviour Research and Therapy, 39, 1411-1427. doi:10.1016/S00057967(00)00107-8. doi: 10.1016/S0005-7967(00)00107-8

Kagan, J., \& Snidman, N. (1991). Infant predictors of inhibited and uninhibited profiles. Psychological Science, 2, 40-44. doi: 10.1111/j.1467-9280.1991.tb00094.x

Kagan J., \& Snidman, N. (1999). Early childhood predictors of adult anxiety disorders. Society for Biological Psychiatry, 46, 1536-1541. doi: 10.1016/S0006-3223(99)00137-7

Kagitcibasi, C. (2010). Self, family, and human development: Cultural psychology. Istanbul: Koc University Press.

Karreman, A., de Haas, S., van Tuijl, C., van Aken, M., \& Dekovic, M. (2010). Relations among temperament, parenting and problem behavior in young children. Infant Behavior and Development, 33, 39-49. doi: 10.1016/j.infbeh.2009.10.008

Kiff, C., Lengua, L., \& Zalevski, M. (2011). Nature and nurturing: Parenting in the context of child temperament. Clinical Child and Family Psychology Review, 14, 251-301. doi: 10.1007/s10567-011-0093-4

Klein, D. M., Dyson, M. W., Kujawa, A. J., \& Kotov, R. (2012). Temperament and internalizing disorders. In M. Zentner \& R. L. Shiner (Eds.), Handbook of temperament (pp. 541-561). New York, NY: Guilford Press.

Kuhlman, K., Olson, S., \& Lopez-Duran, N. (2013). Predicting developmental changes in internalizing symptoms: Examining the interplay between parenting and neuroscience stress reactivity. Developmental Psychobiology, 56, 908-923. doi:10.1002/dev.21166

Lengua, L. J., Wolchik, S., Sandler, I., \& West, S. (2000). The additive and interactive effects of temperament in predicting adjustment problems of children of divorce. Journal of Clinical Child Psychology, 29, 232-244. doi: 10.1207/S15374424jccp2902_9

Letcher, P., Smart, D., Sanson, A., \& Toumbourou, J. (2008). Psychosocial precursors and correlates of differing internalizing trajectories from 3 to 15 years. Social Development, 18, 618-646. doi: 10.1111/j.1467-9507.2008.00500.x

Leve, L., Kim, H., \& Pears, K. (2005). Childhood temperament and family environment as predictors of internalizing and externalizing trajectories from ages 5 to 17. Journal of Abnormal Child Psychology, 33, 505-520. doi: 10.1007/s10802-005-6734-7

Luebbe, A., \& Bell, D. (2014). Positive and negative family emotional climate differences predict youth anxiety and depression via distinct affective pathways. Journal of Abnormal Child Psychology, 42, 897-911. doi: 10.1007/s10802-013-9838-5.

McLeod, B., Weisz, J., \& Wood, J. (2007). Examining the association between parenting and childhood depression: A meta-analysis. Clinical Psychology Review, 27, 968-1003. doi: 10.1016/j.cpr.2007.03.001

Mesman, J., Bongers, I. L., \& Koot, H. M. (2001). Preschool developmental pathways to preadolescent internalizing and externalizing problems. Journal of Child Psychology and Psychiatry, 42, 679-689. doi: 10.1111/1469-7610.00763

Mills, R. S. L., Hastings, P. D., Helm, J., Serbin, L. A., Etezadi, J., Stack, D. M., et al. (2012). Temperamental, parental, and contextual contributors to early-emerging internalizing problems: A new integrative analysis approach. Social Development, 21, 229-253. doi: 10.1111/j.1467-9507.2011.00629.x

Murray, L., Creswell, C., \& Cooper, P. J. (2009). The development of anxiety disorders in childhood: An integrative review. Psychological Medicine, 39, 1413-1423. doi: 10.1017/ S0033291709005157 
Muthadie, L., Zhou, Q., Eisenberg, N., \& Wang, Y. (2013). Predicting internalizing problems in Chinese children: The unique and interactive effects of parenting and child temperament. Developmental Psychopathology, 25, 653-667. doi:10.1017/S0954579413 000084

Nacak, M., Yagmurlu, B., Durgel, E., \& van de Vijver, F. (2011). Parenting in metropolis and Anatolian samples: The role of residence and education in beliefs and behaviors. Turkish Journal of Psychology, 26, 85-100.

Oland, A., \& Shaw, D. (2005). Pure versus co-occurring externalizing and internalizing symptoms in children: The potential role of socio-developmental milestones. Clinical Child and Family Psychology Review, 8, 247-270. doi: 10.1007/s10567-005-8808-z

Oldehinkel, A., Veenstra, R., Ormel, J., de Winter, A., \& Verhulst, F. (2006). Temperament, parenting, and depressive symptoms in a population sample of preadolescents. Journal of Child Psychology and Psychiatry, 47, 684-695. doi: 10.1111/j.1469-7610.2005.01535.x

Operario, D., Tschann, J., Flores, E., \& Bridges, M. (2006). Brief report: Associations of parental warmth, peer support, and gender with adolescent emotional distress. Journal of Adolescence, 29, 299-305. doi: 10.1016/j.adolescence.2005.07.001

Paterson, G., \& Sanson, A. (1999). The association of behavioral adjustment to temperament, parenting and family characteristics among 5 year old children. Social Development, 8, 293-309. doi: 10.1111/1467-9507.00097.

Pfeifer, M., Goldsmith, H., Davidson, R., \& Rickman, M. (2002). Continuity and change in inhibited and uninhibited children. Child Development, 73, 1474-1485. doi: 10.1111/ 1467-8624.00484

Porter, C., Hart, C., Yang, C., Robinson, C., Olsen, S., Zeng, Q., et al. (2005). A comparative study of child temperament and parenting in Beijing, China and the western United States. International Journal of Behavioural Development, 29, 541-551. doi: 10.1177/ 01650250500147402

Rapee, R. (2001). The development of generalized anxiety. In M. Vasey \& M. Dadds (Eds.), The developmental psychopathology of anxiety (pp. 481-503). Cary, NC: Oxford University Press.

Rapee, R., Schniering, C., \& Hudson, J. (2009). Anxiety disorders during childhood and adolescence: Origins and treatment. Annual Reviews in Clinical Psychology, 5, 311-341. doi: 10.1146/annurev.clinpsy.032408.153628

Raudino, A., Murray, L., Turner, C., Tsampala, E., Lis, A., de Pascalis, L., et al. (2013). Child anxiety and parenting in England and Italy: The moderating role of maternal warmth. Journal of Child Psychology and Psychiatry, 54, 1318-1326. doi:10.1111/ jcpp. 12105

Sagrestano, L., Paikoff, R., Holmbeck, G., \& Fendrich, M. (2003). A longitudinal examination of familial risk factors for depression among inner-city African American adolescents. Journal of Family Psychology, 17, 108-120. doi: 10.1037/0893-3200.17.1.108

Sen, H., Yavuz, H. M., \& Yagmurlu, B. (2013). Parenting: The Turkish context. In. H. Selin (Ed.), Parenting across cultures: Childrearing, motherhood and fatherhood in nonwestern cultures. Netherlands: Springer Publishers.

Siu, A. (2008). A prevalence study on internalizing problems among primary school children in Hong Kong. Journal of Child and Family Studies, 17, 779-790. doi:10.1007/s10826008-9189-y

Stifter, C., Putnam, S., \& Jahromi, L. (2008). Exuberant and inhibited toddlers: Stability of temperament and risk for problem behavior. Developmental Psychopathology, 20, 401421. doi: 10.1017/S0954579408000199

Van der Bruggen, C., Stams, G., \& Bogels, S. (2008). Research review: The relation between child and parent anxiety and parental control: A meta-analytic review. Journal of Child Psychology and Psychiatry, 49, 1257-1269. doi: 10.1111/j.14697610.2008.01898.x

Von Suchodoletz, A., Trommsdorff, G., \& Heikamp, T. (2011). Linking maternal warmth and responsiveness to children's self-regulation. Social Development, 20, 486-503. doi: $10.1111 / \mathrm{j} .1467-9507.2010 .00588 . x$

Wei, C., \& Kendall, P. (2014). Parental involvement: Contribution to childhood anxiety and its treatment. Clinical Child and Family Review, 17, 319-339. doi: 10.1007/s10567-014$0170-6$ 
128 H. Melis Yavuz, Bilge Selcuk, Feyza Corapci et al.

Wilmshurst, L. (2009). Anxiety disorders with likely onset in childhood. Abnormal child psychology: A developmental perspective (pp. 195-222). New York, NY: Taylor \& Francis Group.

Wood, J., McLeod, B., Sigman, M., Hwang, W. C., \& Chu, B. (2003). Parenting and childhood anxiety: Theory, empirical findings, and future directions. Journal of Child Psychology and Psychiatry, 44, 134-151. doi: 10.1111/1469-7610.00106

Yagmurlu, B., \& Altan, O. (2010). Maternal socialization and child temperament as predictors of emotion regulation in Turkish preschoolers. Infant and Child Development, 19, 275-296. doi: 10.1002/icd.646

Yagmurlu, B., \& Sanson, A. (2009). The role of child temperament, parenting and culture in the development of prosocial behaviors. Australian Journal of Psychology, 61, 77-88. doi: $10.1080 / 00049530802001338$

Zhou, Q., Lengua, L., \& Wang, Y. (2009). The relations of temperament reactivity and effortful control to children's adjustment problems in China and the United States. Developmental Psychology, 45, 724-739. doi: 10.1037/a0013776 